\title{
Adquisició del llenguatge i comprensió emocional en infants amb sordesa
}

\author{
Francesc Sidera, Georgina Perpiñà, Elisabet Serrat \\ Departament de Psicologia, Universitat de Girona \\ francesc.sidera@udg.edu \\ georgina.perpinya@udg.edu \\ elisabet.serrat@udg.edu
}

Recepció: 12/05/2020, acceptació: 01/09/2020

Resum: La majoria d'infants amb sordesa fills de pares oients presenten mancances en l'àmbit lingüístic i comunicatiu, a causa d'un possible accés limitat al llenguatge i a un entorn conversacional empobrit. Aquestes dificultats lingüístiques i comunicatives dels infants amb sordesa, que poden existir malgrat la utilització d'ajudes auditives, poden repercutir en diversos aspectes del seu desenvolupament. En particular, poden influir negativament en la seva capacitat de comprendre les emocions pròpies i alienes, i en conseqüència, també el seu funcionament social, el seu benestar i l'èxit acadèmic. En aquesta línia, aquest article se centra en intentar explicar de quina manera les diferents característiques de la sordesa i del seu entorn, així com de la interacció entre ambdós, poden implicar deficiències en l'input lingüístic i conversacional que afecten les seves habilitats lingüístiques i comunicatives. A la vegada, pretenem mostrar com les mancances en aquestes habilitats lingüístiques poden implicar dificultats en diferents aspectes de la comprensió emocional dels infants amb sordesa, que poden allargar-se fins a l'etapa adulta. A més, es revisen algunes investigacions que aporten idees rellevants sobre com millorar la capacitat de comprensió emocional dels infants amb sordesa, que se centren sobretot en la utilització d'estratègies conversacionals per ajudar els infants a comprendre les emocions de les persones en diferents situacions.

Paraules clau: comprensió emocional, infants, sordesa, adquisició del llenguatge, habilitats comunicatives i conversacionals, intervenció.

\section{Adquisición del lenguaje y comprensión emocional en niños y niñas con sordera}

Resumen: La mayoría de los niños y niñas con sordera hijos de padres oyentes presentan carencias a nivel lingüístico y comunicativo, a causa de un posible acceso limitado al lenguaje y a un entorno conversacional empobrecido. Estas dificultades lingüísticas y comunicativas de los niños y niñas sordos, que pueden existir a pesar de la utilización de ayudas auditivas, pueden repercutir en varios aspectos de su desarrollo. En particular, pueden influir negativamente en su capacidad de comprender las emociones propias y ajenas, y en consecuencia, también a su funcionamiento social, su bienestar, y el éxito académico. En esta línea, este artículo se centra en intentar explicar de qué manera las diferentes características de la sordera y de su entorno, así como de la interacción entre ambos, pueden implicar deficiencias en el input lingüístico y conversacional que 
afectan a sus habilidades lingüísticas y comunicativas. A su vez, se pretende mostrar como las carencias en estas habilidades lingüísticas pueden implicar dificultades en diferentes aspectos de la comprensión emocional de los niños y niñas con sordera, que pueden alargarse hasta la etapa adulta. Además, se revisan algunas investigaciones que aportan ideas relevantes sobre cómo mejorar la capacidad de comprensión emocional de los niños y niñas con sordera, que se centran sobre todo en la utilización de estrategias conversacionales para ayudar a los niños y niñas a comprender las emociones de las personas en diferentes situaciones.

Palabras clave: comprensión emocional, niños, niñas, sordera, adquisición del lenguaje, habilidades comunicativas y conversacionales, intervención.

\section{Language acquisition and emotional comprehension in children with deafness}

Abstract: Most children with deafness born to hearing parents have language and communication impairments, as a result of a possible limited access to language and to an impoverished conversational environment. These linguistic and communicative difficulties, which may exist despite the use of hearing aids, can affect various developmental aspects of children with deafness. In particular, these difficulties may negatively affect their ability to understand their own and others' emotions, and consequently, their social functioning, their well-being and their academic success. Thus, this paper focuses on trying to explain how the different characteristics of deafness and environment, as well as the interaction between the two, can lead to deficiencies in linguistic and conversational input that affect children's language and communication skills. At the same time, we aim to show how deficiencies in these linguistic skills can lead to difficulties in different aspects of deaf children's emotional comprehension, which can extend into adulthood. In addition, we review some research that provides relevant ideas on how to improve deaf children's emotional understanding, which focuses mainly on the use of conversational strategies to help children understand people's emotions in different situations.

Keywords: emotion understanding, children, deafness, language acquisition, communicative and conversational abilities, intervention.

\section{INTRODUCCIÓ}

La comprensió emocional fa referència al coneixement que les persones tenim sobre les emocions pròpies i dels altres. Aquesta comprensió inclou la capacitat de reconèixer, regular i amagar les emocions, així com de comprendre'n les seves causes, o la relació entre les emocions i els nostres desitjos o creences (Pons, Harris i de Rosnay 2004). Aquesta capacitat de comprendre les emocions està vinculada a diversos aspectes del funcionament social, del benestar psicològic i de l'èxit acadèmic (Pons et al. 2019), i les dificultats amb la comprensió emocional estan associades a problemes de comportament i fins i tot a trastorns mentals (Kårstad, Wichstrøm, Reinfjell, Belsky i Berg-Nielsen 2015). És, per tant, una capacitat molt important en el desenvolupament infantil. Com argumentarem al llarg de l'article, el desenvolupament de la comprensió emocional pot estar afectat en els infants amb sordesa, com a conseqüència de possibles limitacions en el desenvolupament lingüístic i comunicatiu. Així doncs, a continuació s'explicarà amb més detall què és la comprensió emocional i com es relaciona amb el desenvolupament del 
llenguatge; seguidament, ens centrarem en les possibles dificultats lingüístiques dels infants amb sordesa; després, revisarem les dificultats de comprensió emocional en aquests infants i, finalment, apuntarem a algunes possibilitats per millorar la seva capacitat de comprensió emocional.

\section{DESENVOLUPAMENT DEL LLENGUATGE I COMPRENSiÓ EMOCIONAL}

Segons Pons et al. (2004) la comprensió emocional està dividida en tres nivells, de dificultat progressiva. El primer, fa referència a aquells aspectes més externs de les emocions, i es desenvolupa caps als cinc anys. Dins d'aquest primer nivell s'inclouen components com el reconeixement i la identificació de les emocions, la comprensió de l'afectació que tenen les causes externes en les emocions dels altres (per exemple, un nen comprèn que el seu amic està trist per haver perdut la seva joguina preferida) o el paper que té el record en les emocions. El segon nivell se centra en els aspectes mentals de les emocions. Així doncs, en aquest nivell els infants entenen que les reaccions emocionals depenen dels desitjos i que aquests no són iguals per a tothom, que les creences de cadascú (allò que sabem o pensem sobre la realitat) determinen la seva reacció emocional davant d'una situació, o que existeixen emocions ocultes (l'existència d'una discrepància entre l'expressió emocional mostrada i la sentida realment). Finalment, el tercer nivell té en compte els aspectes més reflexius de les emocions i els infants comencen a adonar-se de l'existència de sentiments ambivalents (és a dir, que una persona pot tenir emocions contradictòries davant d'una mateixa situació), d'estratègies de control cognitiu de les emocions i de la relació existent entre emoció i moralitat (els sentiments negatius provenen d'una acció moralment reprovable, com mentir o robar, mentre que els sentiments positius, d'accions lloables).

Aquests tres nivells es regeixen per una organització causal: entendre aspectes externs de les emocions (primer nivell) és un prerequisit per entendre aspectes més psicològics d'aquestes emocions (segon nivell) i, alhora, entendre aquests aspectes psicològics és fonamental per comprendre els aspectes reflexius en les emocions (tercer nivell) (Pons et al. 2004).

Harris, Rosnay i Pons (2005) parlen de la relació entre desenvolupament del llenguatge i comprensió emocional, afirmant que el llenguatge és fonamental pel desenvolupament de la comprensió d'estats mentals i emocionals. En el seu estudi posen de manifest que els infants amb més habilitats lingüístiques entenen més els estats mentals, de la mateixa manera que l'accés i l'exposició a converses riques amb paraules referides a estats mentals i emocionals és un predictor potent que promou la comprensió d'aquests estats. Altres autors que han estudiat la relació entre el desenvolupament lingüístic i el desenvolupament emocional han estat Taumoepeau i Ruffman (2006, 2008), afirmant que caps als 30 mesos es produeix un gran augment en l'adquisició de vocabulari relacionat amb les emocions. D'altra banda, De Stasio, Fiorilli i Di Chiacchio (2014) han trobat associacions entre els components més bàsics de la comprensió emocional (identificació d'expressions facials, comprensió de la causalitat i comprensió de desitjos) i el nivell de gramàtica en infants de 3 a 6 anys. Això concorda amb Sidera, Serrat i Amadó (2014), que mostren que la comprensió d'emocions, en aquest cas fingides, està relacionada amb el vocabulari i la gramàtica. En resum, tant el vocabulari, com determinats aspectes sintàctics, pragmàtics i conversacionals es relacionen directament amb una millor habilitat mentalista (Serrat, Sidera, Rostan i Amadó 2015). 
Avui en dia hi ha debat sobre els mecanismes a partir dels quals el llenguatge i la conversa ajuden a la comprensió dels estats mentals. Bàsicament, hi ha tres interpretacions compatibles entre si.

Una primera és la de l'enriquiment lèxic, que defensa que els guanys en la comprensió emocional són deguts a l'adquisició de vocabulari d'estats mentals. Des d'aquesta visió, entre els dos i els dos anys i mig els infants ja utilitzen algunes paraules per referir-se tant a les emocions negatives com a les positives (content, trist, enfadat, por...) i a partir dels tres anys, es fa evident la seva habilitat per comentar situacions relacionades amb l'emoció. Aquests assoliments permeten que, progressivament, puguin expressar i comprendre les emocions pròpies i dels altres de manera més precisa, complexa i clara (v. Serrat i Sidera 2018).

Una segona interpretació és la de l'enriquiment sintàctic. Des d'aquesta perspectiva, els guanys en la comprensió d'estats mentals són fruit de l'adquisició d'eines sintàctiques que permeten als infants integrar un pensament dins un altre. En aquest cas, el llenguatge té un paper representacional (de Rosnay, Pons, Harris i Morrell 2004). S'ha demostrat que dominar determinades estructures sintàctiques (com per exemple les oracions completives) pot ser útil per comprendre determinats estats mentals (v. de Villiers i de Villiers 2000). Així, per exemple, la frase «en Joan pensa que dins la capsa hi ha caramels» (quan en realitat hi ha pedres) permet representar el fet que en Joan té una creença falsa sobre el contingut de la capsa.

Finalment, una última visió sobre com el llenguatge ajuda a entendre els significats dels estats mentals la trobem en l'enriquiment pragmàtic. Aquesta defensa que els beneficis provenen de les converses en què hi ha diverses perspectives sobre una mateixa realitat. En aquest sentit, la interacció lingüística es considera un factor essencial perquè els nens extreguin el significat de les paraules i les expressions en les converses i jocs (v. Levy i Nelson 1994, Montgomery 2005, Nelson 2007, Ornaghi, Brockmeier i Grazzani Gavazzi 2011, Siegal 2008). De la mateixa manera, el fet d'expressar l'existència de diferents perspectives individuals sobre un mateix fet ajuda a l'adquisició de la comprensió d'emocions (Morra, Parrella i Camba 2011).

Finalment, s'ha demostrat també que existeixen diferències individuals pel que fa a la comprensió emocional dels infants i que aquestes diferències són estables i observables des de la primera infància (Pons i Harris 2005). El contingut de les interaccions amb els pares i la pròpia motivació per categoritzar els estats mentals fruit de les converses espontànies són els causants d'aquestes primeres diferències. En aquest sentit, és evident que aquells infants que creixen en entorns on es parla obertament de les emocions i on es produeixen converses amb alta càrrega emocional tenen avantatge sobre aquells que no tinguin accés a aquest tipus d'interaccions (Harris, de Rosnay i Pons 2016), ja que un context propici com el descrit ajuda al fet que el nen tingui un coneixement emocional més ben definit, i per tant també una major capacitat per entendre les complexitats de les emocions en diferents situacions (Lindquist, MacCormack i Shablack 2015). A continuació, ens centrarem en les possibles dificultats lingüístiques i comunicatives dels infants amb sordesa, que els pot afectar la comprensió emocional.

\section{DESENVOLUPAMENT DEL LLENGUATGE I SORDESA}

Els infants adquireixen una llengua gràcies a posar en marxa les seves capacitats cognitives en contextos comunicatius en què s'utilitza un sistema simbòlic i estructural. En aquest sentit, i de manera molt sintètica, podem dir que dues forces principals guien el desenvolupament d'una primera llengua: l'habilitat dels infants per descobrir significats a

Adquisició del llenguatge i comprensió emocional en infants amb sordesa Francesc Sidera, Georgina Perpiñà i Elisabet Serrat (2020) Llengua, Societat i Comunicació, núm. 18 http://revistes.ub/index.php/LSC / lsc@ub.edu http://creativecommons.org/licenses/by-nc-nd/4.o 
partir de l'input que tenen a disposició, i l'input que proporcionen els parlants que interactuen amb els infants en contextos de parla significatius. D'una banda, tenim les habilitats cognitives pròpies dels infants per extreure significats i estructures a partir de l'input proporcionat pels pares i les mares (Feijóo, Muñoz, Amadó i Serrat 2017), així com les habilitats sociocognitives de lectura d'intencions (Tomasello 2000). D'altra banda, hi ha la bastida que proporcionen les mares i els pares amb la parla que utilitzen amb els seus fills en contextos d'interacció comunicativa, la qual proporciona suport i oportunitats múltiples per aprendre la llengua (Rowe 2012). Les habilitats cognitives dels infants i la interacció de l'entorn per donar lloc al desenvolupament comunicatiu, cognitiu i afectiu dels infants són dos factors en interacció constant. Tot i que podem destriar-los, no són independents i si algun d'aquests es troba alterat hi haurà repercussions en l'altre, tal com pot observar-se en el desenvolupament dels infants amb sordesa.

Al final del primer any de vida els infants amb un desenvolupament típic han descobert patrons de significat en la parla o en el flux de signes visuals (Levine, StrotherGarcia, Golinkoff i Hirsh-Pasek 2016). És a dir, durant el primer i segon any de vida, es constitueixen les bases del desenvolupament del llenguatge, ja sigui oral o signat. En aquests moments, els infants comencen a descobrir connexions entre el llenguatge i el món. Si aquesta fonamentació no hi és, el desenvolupament lingüístic es veurà afectat, i també d'altres desenvolupaments que hi estan relacionats $\mathrm{i}$ aprenentatges posteriors (Lederberg, Schick i Spencer 2013).

Les habilitats pròpies dels infants i els efectes de l'entorn entraran en joc des dels inicis del desenvolupament, de manera que en els infants oients, el desenvolupament del llenguatge comença tan aviat com el sistema auditiu del fetus és capaç de percebre la veu de la seva mare (els últims tres mesos d'embaràs). Mentre que en el cas dels nens amb sordesa nascuts de famílies signants, el desenvolupament del llenguatge començarà després del naixement, de seguida que l'infant pugui percebre els signes. Hi ha diversos estudis que han mostrat que hi ha una gran similitud entre les etapes d'adquisició de la competència lingüística en els infants amb sordesa nascuts de pares amb sordesa (en llengua de signes) i les etapes del desenvolupament de la mateixa competència en els nens oients (llengua oral) de famílies oients (Newport i Meier 1985; Schick, Marschark i Spencer 2006). El que mostren aquests estudis és que les fites en el desenvolupament del llenguatge s'assoleixen a la mateixa edat, independentment de la modalitat de llenguatge, tant pel que fa al vocabulari i la gramàtica inicials, com pel que fa al discurs narratiu i conversacional, només amb algunes diferències derivades de la modalitat (Tomaszewski, Krzysztofiak i Moron 2019).

Ara bé, la gran majoria de nens amb sordesa neixen en llars amb pares oients (un 94\% segons Skliar, Masone, Veinberg 1995) i independentment de la modalitat dominant, és probable que tinguin un accés limitat al llenguatge durant el primer període de les seves vides. Pot ser així tant pel que fa al temps que poden tardar a rebre ajudes auditives (Mauldin 2012) com per l'absència de models lingüístics fluids (Koulidobrova, Kuntze i Dostal 2018, Mitchell i Karchmer 2015). En definitiva, quan l'entorn no pot oferir un sistema lingüístic a l'infant amb sordesa, aquests infants veuen compromès el seu procés d'adquisició del llenguatge (Knoors 2016, Scott i Dostal 2019). En el cas dels infants amb sordesa amb pares oients que no saben llengua de signes, l'inici de l'adquisició del llenguatge pot endarrerir-se de manera important, tot i que aquests infants podrien adquirir simultàniament una llengua de signes i una llengua oral, o bé adquirir una llengua de signes amb d'altres persones o en un altre entorn -veure Humphries et al. (2014) per a una ampliació d'aquests aspectes. 
Com hem comentat, els fonaments de l'adquisició d'una primera llengua es donen durant els primers anys de vida. En aquest sentit, si un infant no està exposat a una llengua accessible de manera regular i freqüent durant els primers anys, és poc probable que utilitzi una llengua amb una fluïdesa similar a la d'un nadiu (Mayberry 1998). Aquest període, conegut com el període crític en l'adquisició del llenguatge, es pot aplicar tant al desenvolupament de la llengua oral com al desenvolupament de la llengua de signes (Tomaszewski et al. 2019). Segons Humphries et al. (2014), els estudis sobre infants amb sordesa que no van rebre una llengua accessible fins després d'un període sensible degut a la carència d'ajudes auditives o perquè se'ls nega la llengua signada, mostren una reduïda facilitat per al llenguatge. En la mateixa línia, també s'han adduït repercussions posteriors tant en l'àmbit cognitiu com en l'àmbit de la salut mental (Hall 2017). Ara bé, podria considerar-se que actualment els infants amb sordesa nascuts de famílies oients tenen a disposició un sistema lingüístic molt aviat en el seu desenvolupament. És així perquè la possibilitat d'accés a ajudes auditives permetria l'accés al llenguatge oral d'una manera que no era possible fa uns anys. Per tant, podria assumir-se que no hi ha d'haver repercussions en el seu desenvolupament lingüístic i comunicatiu.

Per aprofundir en aquesta darrera qüestió, en aquest article ens centrem principalment en infants que encara no han adquirit el llenguatge (amb sordesa prelocutiva) i en la intensitat de la pèrdua auditiva com a factor central, i considerant que hi ha accés a dispositius tecnològics d'ajuda auditiva (com els audiòfons i els implants coclears -a partir d'ara IC). Molt breument revisarem alguns estudis sobre infants amb sordesa lleugera o moderada i estudis sobre infants amb sordesa severa o profunda, nascuts de famílies oients - una explicació sobre tipus de sordesa i graus de pèrdua auditiva pot trobar-se a Llombart (n.d.).

Diversos estudis mostren que, com a grup, els infants amb sordesa lleugera o moderada tendeixen a mostrar endarreriment o desviació en el desenvolupament de la fonologia, el vocabulari i la morfosintaxi, però no en l'adquisició de les habilitats lectores (Moeller, Tomblin, Yoshinaga-Itano, Connor i Jerger 2007, Tuller i Delage 2014). A l'estudi de Halliday, Tuomainen i Rosen (2017) també s'observa que les habilitats lingüístiques dels infants amb sordesa lleugera o moderada són més pobres en comparació amb el grup d'infants oients, però sovint dins dels límits normals per a la seva edat. Aquests autors remarquen el que altres estudis també havien observat, que hi ha diferències individuals importants, amb alguns infants dins dels límits de la normalitat $\mathrm{i}$ d'altres que mostren evidència de llenguatge oral i escrit amb dificultats clínicament significatives.

Per la seva banda, els infants amb pèrdua auditiva severa o profunda (amb IC), independentment de quina habilitat lingüística s'examini, també mostren un nivell per sota dels infants de la seva mateixa edat sense sordesa, pel que fa al vocabulari (Conway, Pisoni, Anaya, Karpicke i Henning 2011), la gramàtica (Tobey et al. 2013) o la fonologia (Geers i Hayes 2011). A partir de la revisió de diversos estudis, Nittrouer i Caldwell-Tarr (2016) conclouen que, des d'una visió optimista, aproximadament la meitat dels infants amb aquest nivell de pèrdua auditiva $\mathrm{i}$ amb implant coclear se situa en el rang normal d'habilitats per a la seva edat, cosa que suposa un resultat molt bo si tenim en compte els resultats que s'observaven abans dels implants coclears. Ara bé, els mateixos autors, apunten que la meitat dels infants amb una pèrdua d'audició severa o profunda (amb IC) mostren habilitats lingüístiques en el percentil 15 de la classificació d'infants sense dificultats auditives. Per tant, tot i els IC, els infants amb sordesa severa o profunda 
encara no arriben als nivells d'habilitat lingüística que podrien abastar si no tinguessin sordesa.

Així doncs, els estudis amb infants amb sordesa fills de pares oients i no signants, assenyalen que la sordesa en aquest grup d'infants, independentment de la intensitat de la pèrdua auditiva i de les ajudes auditives, comporta dificultats en l'adquisició d'una primera llengua (Halliday et al. 2017, Nittrouer i Caldwell-Tarr 2016). A més, cal afegir que aquestes dificultats poden afectar negativament el desenvolupament cognitiu d'aquests infants (Hall, Eigsti, Bortfeld i Lillo-Martin 2017, Hall, Levin i Anderson 2017). Com sostenen Lederberg et al. (2013) no hi ha evidència que els infants no puguin aprendre una llengua a partir de múltiples modalitats o que utilitzar una llengua visual afecti el desenvolupament d'una llengua oral, però sí que hi ha evidència que no tenir accés a una llengua té efectes desenvolupamentals negatius a llarg termini.

També, els entorns comunicatius i lingüístics poden variar pel que fa a la riquesa conversacional, i encara més en els infants amb sordesa, en la mesura que potencialment la comunicació amb els cuidadors pot ser més reduïda o de menor qualitat, fet que pot ser particularment important per al desenvolupament comunicatiu i lingüístic (Desjardin i Eisenberg 2007, Fagan, Bergeson i Morris 2014) i, a partir d'aquí, per al seu desenvolupament en general. A continuació, ens centrarem en les conseqüències que poden haver-hi, concretament, pel que fa a la comprensió emocional.

\section{COMPRENSIÓ EMOCIONAL I LLENGUATGE EN INFANTS AMB SORDESA}

Diverses investigacions suggereixen que els infants amb sordesa fills de pares oients no signants poden tenir dificultats de teoria de la ment, és a dir, de comprendre els estats mentals dels altres, com ara els desitjos, les creences o les intencions, i en particular les emocions (Peterson 2016, Peterson, O’Reilly i Wellman 2016, Wellman 2018). Per tant, les dificultats de comprensió emocional dels infants sords, en les quals ens centrarem en aquest apartat, s'han d'emmarcar en unes dificultats més generals que poden tenir a l'hora de comprendre els estats mentals i els comportaments de les persones. Tot i que aquí ens centrarem en la comprensió emocional, cal tenir en compte que aquesta comprensió no està deslligada de la comprensió d'altres estats mentals com ara els desitjos i les creences, tal com hem explicat a l'apartat 2.

Alguns investigadors han suggerit que la capacitat dels pares i les mares de comunicar-se amb els infants a través d'una llengua de signes des del naixement (com és el cas dels infants amb sordesa, fills de pares que també tenen sordesa) és crucial per al desenvolupament de les capacitats sociocognitives (entre les quals trobem la comprensió d'emocions). Tot $\mathrm{i}$ això, fins $\mathrm{i}$ tot aquests infants poden tenir dificultats en el desenvolupament de les capacitats sociocognitives si s'escolaritzen en escoles que són de modalitat oral. Per tant, són factors importants en el desenvolupament de la capacitat dels infants de comprendre els estats mentals i emocionals, no només l'accés, com a mínim, a una primera llengua al més aviat possible, ja sigui oral o signada, sinó també el fet de poder-la utilitzar per parlar sobre els pensaments i sentiments dels altres tant a casa com a l'escola (Meristo et al. 2007, Peterson 2009).

Les dificultats dels infants sords de comprendre els estats mentals i emocionals els poden crear fàcilment malentesos en les seves interaccions comunicatives, i fins i tot poden explicar alguns problemes de comportament detectats en els infants amb sordesa, com per exemple la desconfiança vers els altres (Rieffe i Meerum Terwogt 2000). Pel que fa a les dificultats de comprensió emocional en particular, aquestes refereixen a diversos

Adquisició del llenguatge i comprensió emocional en infants amb sordesa Francesc Sidera, Georgina Perpiñà i Elisabet Serrat (2020)

Llengua, Societat i Comunicació, núm. 18 http://revistes.ub/index.php/LSC / lsc@ub.edu 
aspectes, com per exemple a la capacitat de reconèixer facialment les emocions dels altres (Sidera et al. 2017), de comprendre les causes de les emocions (Rieffe, Terwogt, i Smit, 2003), de comprendre les emocions fingides (Sidera, Morgan i Serrat, 2020) o d'atribuirse emocions morals a si mateixos (Hao i Wu 2019). A més, s'han trobat endarreriments en la comprensió emocional també amb infants amb implant coclear i utilitzant tasques noverbals (Wang, Su, i Yan 2016, Wiefferink, Rieffe, Ketelaar, De Raeve i Frijns 2013). Tot i que els implants coclears existents avui solen permetre un bon reconeixement de la parla, i que l'edat d'implantació és clau en el desenvolupament de la comprensió emocional (Mancini et al. 2016), aquests implants encara tenen algunes mancances en el processament del to de veu i la prosòdia. Així doncs, el processament de les emocions i la comprensió emocional a través de la veu estan afectats en els infants i adults amb implant coclear. De fet, el to de veu és important en la interpretació del significat dels missatges de manera especial en algunes llengües, com ara el xinès, i per tant aquesta interpretació es pot veure encara més afectada en les persones amb implant coclear parlants d'aquestes llengües (Wang, Wang i Hu 2019).

Tot i que algunes investigacions no han trobat diferències entre infants amb sordesa $i$ oients en alguns aspectes de la comprensió emocional (per exemple: Ziv, Most i Cohen 2013), és possible que alguns d'aquests estudis no hagin detectat dificultats perquè ja han assolit el nivell dels oients en el moment de l'avaluació (Laugen, Jacobsen, Rieffe i Wichstrøm 2017). En aquest sentit, Sidera et al. (2017) trobaren que els infants amb sordesa de la seva mostra no tenien dificultats en el reconeixement facial d'aquelles emocions bàsiques l'etiqueta de les quals s'adquireix abans en el desenvolupament (alegria, tristesa o enuig), però sí en aquelles d'adquisició més tardana (por, sorpresa o fàstic). D'aquesta manera, tot i que les persones amb sordesa continuen desenvolupant les seves capacitats de comprensió d'estats mentals i emocionals fins a l'etapa adulta, hi ha evidències que aquestes capacitats poden seguir endarrerides en l'adultesa (O'Reilly, Peterson i Wellman 2014).

S'ha suggerit que les dificultats en la comprensió emocional dels infants amb sordesa es podrien explicar per les seves dificultats lingüístiques. En aquesta línia, Dyck, Farrugia, Shochet i Holmes-Brown (2004) van trobar que en el seu estudi les dificultats dels infants amb sordesa de reconèixer i comprendre les emocions desapareixien si se'ls comparava amb una mostra d'infants oients amb habilitats lingüístiques similars. No obstant això, els infants oients del seu estudi tenien una edat significativament més gran. En aquest sentit, en l'estudi de Sidera et al. (2017) les diferències en la capacitat de reconèixer emocions facials entre infants amb sordesa i sense es mantingueren significatives malgrat igualar-los amb habilitats lingüístiques, edat i habilitats cognitives (raonament espacial). Aquestes dades podrien justificar la possibilitat que les dificultats de comprensió emocional del infants amb sordesa no s'expliquin només per les seves dificultats en les habilitats lingüístiques formals, sinó que, d'acord amb la hipòtesi conversacional, la privació conversacional i comunicativa en edats primerenques hi podria tenir un paper important (Peterson i Siegal 1995).

D'acord amb aquesta hipòtesi conversacional, els progenitors oients dels infants amb sordesa tendeixen a comunicar-s'hi només sobre temes que tenen una referència visual, $\mathrm{i}$ per tant acaben compartint amb els seus infants menys informació sobre els estats mentals i emocionals de les persones, restringint les experiències dels infants al món mental dels altres, així com el seu accés a vocabulari referit a estats mentals. Això no obstant, tot i que s'ha observat que la participació dels infants en converses referides a estats mentals ajuda el desenvolupament de la comprensió dels estats mentals de les 
persones (Ornaghi et al. 2011), no hi ha evidències que existeixi un període crític en la primera infància en el qual la privació conversacional tingui conseqüències irreparables en la capacitat de comprendre estats mentals i emocionals (v. Peterson et al. 2016). Paral-lelament, el fet que alguns infants amb sordesa i un nivell lingüístic similar al dels infants oients segueixin tenint dificultats amb la comprensió emocional, també es podria explicar perquè han treballat més el llenguatge i no tant la comprensió emocional, i per tant han millorat en uns aspectes però no en els altres.

\section{COM MILLORAR LA COMPRENSIÓ EMOCIONAL EN ELS INFANTS AMB SORDESA?}

En els apartats anteriors hem vist que la comprensió emocional té un paper important en el desenvolupament social i en el benestar dels infants. A més, també hem explicat que aquesta comprensió està influïda per les habilitats lingüístiques, comunicatives i conversacionals dels infants, de manera que aquelles intervencions dirigides a reduir la privació lingüística que tenen alguns infants amb sordesa poden ser eficaces per millorar la seva comprensió emocional. En aquest sentit, alguns autors argumenten que tots els infants sords haurien d'aprendre una llengua de signes (v. Humphries et al. 2014), encara que d'altres autors ho posen en dubte (v. Geers et al. 2017). Ara bé, més enllà de les possibles intervencions dirigides a millorar el nivell lingüístic i comunicatiu dels infants, en les quals no volem entrar en detall en aquesta revisió, la literatura científica aporta algunes idees i estratègies que es poden utilitzar específicament per millorar les capacitats de comprensió emocional. A continuació en farem una breu revisió.

D’una banda, i pel que fa als pares i les mares, Kårstad et al. (2015) trobaren que la seva precisió a l'hora d'estimar la comprensió emocional dels seus fills als quatre anys era un predictor de la capacitat dels infants de comprendre les emocions als sis anys. D'altra banda, a l'estudi de Laugen et al. (2017) van trobar que els progenitors d'infants amb sordesa i audiòfons s'ajustaven millor que els progenitors d'infants oients a les capacitats de comprensió emocional dels seus infants (tot i que ambdós grups tendien a sobreestimar aquestes capacitats), en els quals no es detectaren dificultats en la comprensió emocional. Aquests autors hipotetitzaren que aquestes millors estimacions es podien explicar per les intervencions centrades en les famílies amb els infants amb sordesa. En aquest sentit, ajudar els pares i les mares d'infants amb sordesa a detectar millor les capacitats de comprensió emocional pot ser una via d'intervenció eficaç per millorar-los aquestes capacitats. A part d'això, hi ha evidències que les capacitats sociocognitives dels infants poden millorar entrenant les mares a parlar amb els infants de manera més elaborada sobre els esdeveniments passats en termes d'estats mentals, especialment amb aquells infants amb un vocabulari reduït. Així doncs, millorant les formes en què els progenitors conversen amb els fills pot millorar la capacitat d'aquests de comprendre els estats mentals i emocionals de les altres persones, fent evident a l'infant que diferents persones poden tenir diferents punts de vista sobre un mateix esdeveniment (Taumoepeau i Reese 2013). En aquest sentit, diversos estudis d'entrenament fets amb infants (oients) han mostrat que la comprensió de la ment de les altres persones pot millorar a través d'entrenaments en els quals s'utilitza el llenguatge per ressaltar que diferents persones poden tenir diferents punts de vista sobre una mateixa realitat (Rostan et al. 2014, Serrat et al. 2013), també en els infants amb dificultats lingüístiques (Serrat et al. 2012). A tall d'exemple d'un estudi amb infants amb sordesa, l'entrenament amb bombolles de pensament (representacions visuals dels pensaments de les persones) s'ha 
mostrat efectiu per millorar la seva comprensió dels estats mentals i emocionals (Wellman i Peterson 2013).

En l'àmbit educatiu, alguns investigadors proposen fomentar el diàleg o les converses entre companys sobre alguns aspectes de la comprensió emocional per tal de millorar-la en els infants amb sordesa (Sarmento-Henrique i Giménez-Dasí 2013). En una línia similar, Peskin i Astington (2004), trobaren que per millorar la comprensió dels estats mentals no n'hi ha prou amb escoltar passivament històries, sinó que és necessari que els infants s'impliquin activament en la construcció d'interpretacions mentalistes del comportament dels personatges. Peng, Johnson, Pollock, Glasspool i Hams (1992) mostraren que, concretament, preguntar als infants sobre les emocions dels personatges pot ser útil per ajudar-los a comprendre que a vegades podem tenir emocions contraposades. A més a més, hi ha estudis fets en l'àmbit escolar que mostren que és possible millorar diferents components de la comprensió emocional dels infants en el grup classe a partir de programes educatius basats en el diàleg i duts a terme pels seus mestres habituals (Pons, Harris, i Doudin 2002), fins i tot amb programes curts i que no impliquen la formació dels mestres (Pons et al. 2019). Així doncs, en general, els estudis d'entrenament dirigits a millorar la comprensió emocional són eficaços, especialment si s'ajusten al nivell desenvolupamental dels infants (Sprung, Münch, Harris, Ebesutani i Hofmann 2015). Tot i això, la majoria dels estudis comentats aquí impliquen a infants sense sordesa, i per tant es fa necessari adaptar-los a infants amb sordesa. Per exemple, les intervencions amb els infants amb sordesa i implant coclear haurien de tenir en compte la seva percepció del to de la parla (Wang et al. 2019).

\section{CONCLUSIONS}

En síntesi, la investigació científica ha mostrat que els infants amb sordesa que tenen dificultats lingüístiques i comunicatives poden tenir afectada la seva comprensió emocional. En aquest sentit, és de vital importància que els infants sords puguin adquirir al més aviat possible una primera llengua (en la modalitat que sigui), com a mínim, i que la puguin practicar al màxim possible en un entorn conversacional ric. Sabem també que, afortunadament, la comprensió emocional pot millorar a través d'intervencions educatives, ja sigui en l'àmbit familiar o escolar. A partir de la breu revisió feta en l'apartat anterior, comentarem breument alguns aspectes que pensem que haurien de considerar aquestes intervencions educatives. En primer lloc, és important augmentar el grau de coneixement dels pares i les mares o els educadors sobre el nivell de comprensió emocional dels infants. En segon lloc, els educadors haurien d'implicar els infants en diàlegs que els ajudin a adonar-se que davant d'una mateixa realitat dues persones poden tenir diferents punts de vista i diferents emocions. Per fer-ho, cal fomentar la construcció d'hipòtesis sobre els estats mentals i emocionals, ja siguin de persones reals o de personatges de contes o de vídeos. En definitiva, tot i que ja coneixem alguns elements importants per a la intervenció, encara és necessari dur a terme molta més recerca per tal d'adaptar les intervencions educatives existents a les característiques dels infants amb sordesa.

\section{AGRAÏMENTS}

L'elaboració d'aquest article ha estat possible gràcies al suport del Ministerio de Economía y Competitividad i el Fondo Europeo de Desarrollo Regional (PSI2015-69419R; MINECO-FEDER). 


\section{REFERÈNCIES}

Conway, Cristopher. M.; Pisoni, David B.; Anaya, Esperanza M.; Karpicke, Jennifer; HENNING, SHIRLEY C. (2011). «Implicit sequence learning in deaf children with cochlear implants». Developmental Science, 14(1), 69-82.

DESJARDin, JeAN; Eisenberg, LAURIE S. (2007). «Maternal contributions: Supporting language development in young children with cochlear implants». Ear and Hearing, 28, 456-469.

De Rosnay, Marc; Pons, Francisco; Harris, Paul L.; Morrell, Julia M. B. (2004). «A lag between understanding false belief and emotion attribution in young children: Relationships with linguistic ability and mothers' mental state language». British Journal of Developmental Psychology, 22, 197-218.

De Stasio, Simona; Fiorilli, Caterina; Di Chiacchio, Carlo (2014). «Effects of verbal ability and fluid intelligence on children's emotion understanding». International Journal of Psychology, 49(5), 409-414.

De Villiers, Jill G.; De Villiers, Peter A. (2000). «Linguistic determinism and the understanding of false beliefs». Mitchell, Peter; Kevin J. Riggs (eds.). Children's reasoning and the mind (pp. 191-228). Hove, England: Psychology Press.

Dyck, Murray J.; Farrugia, Charles; Shochet, Ian M.; Holmes-Brown, Martez (2004). «Emotion recognition/understanding ability in hearing or vision-impaired children: Do sounds, sights, or words make the difference?» Journal of Child Psychology and Psychiatry and Allied Disciplines, 45(4), 789-800.

FAGAN MARY K.; BERgESON TONYA R.; Morris Kourtney (2014). «Synchrony, complexity and directiveness in mothers' interactions with infants pre- and post-cochlear implantation». Infant Behavior and Development, 37, 249-57.

Feijóo, Sara; Muñoz, Carmen Amadó, AnNa; Serrat, Elisabet (2017). «When meaning is not enough: Distributional and semantic cues to word categorization in child directed speech». Frontiers in Psychology, 8, 1242.

GEERS, ANN E.; HAYES, HeATHER (2011). «Reading, writing, and phonological processing skills of adolescents with 10 or more years of cochlear implant experience». Ear and Hearing, 32(1), 49S.

Geers, Ann E.; Mitchell, Christine M.; Warner-CzyZ, ANdrea; Wang, Nae-Yuh; EISENBERG, LAURIE S.; CDACI INVESTIGATIVE TEAM. (2017). Early sign language exposure and cochlear implantation benefits. Pediatrics, 140(1).

Hall, Wyatte C. (2017). «What you don't know can hurt you: The risk of language deprivation by impairing sign language development in deaf children». Maternal and child health journal, 21(5), 961-965.

Hall, Matthew L.; Eigsti, Inge-Marie; Bortfeld, Heather; Lillo-Martin, Diane (2017). "Auditory deprivation does not impair executive function, but language deprivation might: Evidence from a parent-report measure in deaf native signing children». The Journal of Deaf Studies and Deaf Education, 22(1), 9-21.

Hall, Wyatte C.; LeVin, Leonard L.; Anderson, Melissa L. (2017). «Language deprivation syndrome: A possible neurodevelopmental disorder with sociocultural origins». Social Psychiatry and Psychiatric Epidemiology, 52(6), 761-776.

Halliday, Lorna F.; TuOMainen, Outi; Rosen, Stuart (2017). «Language development and impairment in children with mild to moderate sensorineural hearing loss». Journal of Speech, Language, and Hearing Research, 60(6), 1551-1567. 
HAO, JIAN; WU, CHUNSHA (2019). "Deaf children's moral behavior, moral reasoning and emotion attribution». Journal of Deaf Studies and Deaf Education, 24(2), 95-103.

Harris, Paul L.; De Rosnay, Marc; Pons, Francisco (2005). «Language and Children's Understanding of Mental Status». Current Directions in Psychological Science, 14(2), 6973 .

Harris, PaUl L.; De Rosnay, Marc; Pons, Francisco (2016). «Understanding emotion». FELdMAN BARRET, Lisa; LeWIS, Michael; M. HAVIland-Jones, JEANNETte (eds.). Handbook of Emotions (3rd ed., pp. 293-306). New York: The Guilford Press.

Humphries, Tom; Kushalnagar, Poorna; Mathur, Gaurav; Napoli, Donna Jo; Padden, CAROL; RATHMANN, Christian (2014). «Ensuring language acquisition for deaf children: What linguists can do». Language, 90(2), e31-e52.

Kărstad, Silja B.; Wichstrøm, Lars; ReinfJell, Trude; Belsky, Jay; Berg-Nielsen, TURID S. (2015). «What enhances the development of emotion understanding in young children? A longitudinal study of interpersonal predictors». British Journal of Developmental Psychology, 33(3), 340-354.

KNOORS, HARRY (2016). «Foundations for language development in deaf children and the consequences for communication choices». The Oxford Handbook of Deaf Studies in Language, 19-31.

Koulidobrova, Elena; Kuntze, Marlon; Dostal, Hannah (2018). «If you use ASL, should you study ESL? Limitations of a modality-b(i)ased policy». Laguage and Public Policy, 2, e99-e126.

Laugen, Nina J; Jacobsen, Karl H.; RiefFe, Carolien; Wichstrøm, LarS (2017). «Emotion understanding in preschool children with mild-to-severe hearing loss». Journal of Deaf Studies and Deaf Education, 22(2), 155-163.

Lederberg, Amy. R.; Schick, Brenda; Spencer, Patricia E. (2013). «Language and literacy development of deaf and hard-of-hearing children: successes and challenges». Developmental Psychology, 49(1), 15.

Levine, Dani; Strother-Garcia, Kristina; Golinkoff, Roberta Michnick; HirshPASEK, KATHY (2016). «Language development in the first year of life: What deaf children might be missing before cochlear implantation». Otology \& Neurotology, 37(2), e56-e62.

LEVY, ElenA; Nelson, Katherine (1994). «Words in discourse: A dialectical approach to the acquisition of meaning and use». Journal of Child Language, 51, 1095-1102.

LiNDQUist, KRISTEN A.; MACCORMACK, JENNIFER K.; SHABLACK, Holly (2015). «The role of language in emotion: Predictions from psychological constructionism». Frontiers in psychology, 6, 444 .

Llombart, CARLes (n.d.). Consultat el 20 de juliol de 2020 des de http://www.xtec.cat/ cllombar/

Mancini, Patrizia; Giallini, Ilaria; Prosperini, Luca; D’Alesssandro, Hilal Dincer; Guerzoni, LeTIZIA; MurRi, Alessandra; ... NicAstri, MARIA (2016). «Level of emotion comprehension in children with mid to long term cochlear implant use: How basic and more complex emotion recognition relates to language and age at implantation». International Journal of Pediatric Otorhinolaryngology, 87, 219-232.

MAULDIN, LAURA (2012). "Parents of deaf children with cochlear implants: A study of technology and community». Sociolology of Health and Illness, 34, 529-543. 
MAYBERRY, RACHEL I. (1998). «The critical period for language acquisition and the deaf child's language comprehension: A psycholinguistic approach». Bulletin d'Audiophonologie, 14, 349-360.

Meristo, Marek; Falkman, Kerstin W.; HJelmquist, Erland; Tedoldi, Mariantonia; SURIAN, LUCA; SIEGAL, MichAEL (2007). "Language access and theory of mind reasoning: evidence from deaf children in bilingual and oralist environments». Developmental Psychology, 43(5), 1156-1169.

Mitchell, Ross E.; KARChMER, Michaela A. (2015). «Parental hearing status and signing among deaf and hard of hearing students». Sign Language Studies, 5, 231-244.

Moeller, Mary Pat; Tomblin, J. Bruce; Yoshinaga-Itano, Christine; Connor, Carol MCDONALD; JERGER, SUSAN (2007). «Current state of knowledge: Language and literacy of children with hearing impairment». Ear and Hearing, 28(6), 740-753.

Montgomery, Derek E. (2005). «The developmental origins of meaning for mental terms». Astington, JANET WiLdE; BAIRD, JodIE A. (eds.). Why language matters for theory of mind (pp. 106-122). Oxford, United Kingdom: Oxford University Press.

Morra, SERgio; PARrella, Ilaria; CAMba, Roberta (2011). «The role of working memory in the development of emotion comprehension». British Journal of Developmental Psychology, 29(4), 744-764.

NELSON, KATHERINE (2007). «Young minds in social worlds: Experience, meaning, and memory». Cambridge, MA: Harvard University Press.

NEWPORT, ElisSA L.; MEIER, RichaRd (1985). «Acquisition of American Sign Language». Slobin, D. I. (ed.). The Cross-Linguistic Study of Language Acquisition (pp. 881-938). Hillsdale, NJ: Lawrence Erlbaum Associates.

NitTrouer, Susan; CAldwell-TaRr, Amanda (2016). «Language and Literacy Skills in Children with Cochlear Implants: Past and Present Findings». Young, NANCY M.; ILER KIRK, KAREN (eds.). Pediatric Cochlear Implantation (pp. 177-197). Springer

O’Reilly, Karin; Peterson, Candida C.; Wellman, Henry M. (2014). «Sarcasm and advanced theory of mind understanding in children and adults with prelingual deafness». Developmental Psychology, 50(7), 1862-1877.

Ornaghi, Veronica; Brockmeier, Jens; GrazZani Gavazzi, Ilaria (2011). «The role of language games in children's understanding of mental states: A training study». Journal of Cognition and Development, 12(2), 239-259.

Peng, Manli; Johnson, Carl; Pollock, John; Glasspool, Rosalind; Hams, Paul (1992). «Training young children to acknowledge mixed emotions». Cognition and Emotion, 6(5), 387-401.

Peskin, JoAn; Astington, Janet Wilde (2004). «The effects of adding metacognitive language to story texts». Cognitive Development, 19(2), 253-273.

Peterson, CANDida C. (2009). «Development of social-cognitive and communication skills in children born deaf». Scandinavian Journal of Psychology, 50(5), 475-483.

Peterson, CANDida C. (2016). «Empathy and theory of mind in deaf and hearing children». Journal of Deaf Studies and Deaf Education, 21(2), 141-147.

Peterson, Candida C.; O’reilly, K.; Wellman, Henry M. (2016). «Deaf and hearing children's development of theory of mind, peer popularity, and leadership during middle childhood». Journal of Experimental Child Psychology, 149, 146-158. 
Peterson, Candida C.; Siegal, Michael (1995). «Deafness, conversation and theory of mind». Journal of Child Psychology and Psychiatry, 36(3), 459-474.

Pons, Francisco; Giménez-Dasí, Marta; Daniel, Marie-France; Auriac-Slusarczyk, EmmanuÈle; Businaro, Nicoletta; Viana, Karine (2019). "Impact of a low-cost classroom dialogue-based intervention on preschool children's emotion understanding». European Early Childhood Education Research Journal, 27(5), 630-646.

Pons, Francisco; Harris, PaUl L.; De Rosnay, MarC (2004). «Emotion comprehension between 3 and 11 years: Developmental periods and hyerarchical organizations». European Journal of Developmental Psycology, 1(2), 127-152.

Pons, Francisco; Harris, PaUl L. (2005). «Longitudinal change and longitudinal stability of individual differences in children's emotion understanding». Cognition \& Emotion, 19(8), 1158-1174.

Pons, Francisco; Harris, Paul L.; Doudin, Pierre-André (2002). «Ensenyar a Comprendre les Emocions», Suports, 7, 44-53.

Rieffe, Carolien; Terwogt, Mark Meerum (2000). Deaf children's understanding of emotions: «Desires take precedence». Journal of Child Psychology and Psychiatry and Allied Disciplines, 41(5), 601-608.

Rieffe, Carolien; Terwogt, Mark Meerum; Smit, Cootje (2003). «Deaf Children on the Causes of Emotions». Educational Psychology, 23(2), 159-168.

Rostan, Carles; Sidera, Francesc; Serrano, JÈssica; Amadó, Anna; Vallès-Majoral, EDUARD; ESTEBAN, MoISĖs; SERRAT, EliSABET (2014). «Favorecer el desarrollo de la teoría de la mente. Efectos a corto y medio plazo de un entrenamiento en comprensión de la falsa creencia». Infancia y Aprendizaje, 37(3), 498-529.

Rowe, Meredith L. (2012). "A longitudinal investigation of the role of quantity and quality of child-directed speech in vocabulary development». Child Development, 83(5), 1762-1774.

Sarmento-Henrique, Renata; Giménez-Dasí, Marta (2013). «Emotional development of deaf children: a proposal for intervention». International Journal on Mental Health and Deafness, 3(1), 1.

Schick, Brenda; Marschark, Marc; Spencer, Patricia Elisabeth (eds.). (2006). «Advances in the Sign Language Development of Deaf Children». New York, NY: Oxford University Press.

ScotT, Jessica A.; Dostal Hannah M. (2019). «Language Development and Deaf/Hard of Hearing Children». Education Sciences, 9, 135.

Serrat, Elisabet; Rostan, Carles; Vallès-Majoral, Eduard; Esteban, Moisès; Sidera, FRANCESC; SERRANO, JĖSSICA (2013). "Labelling improves false belief understanding. A training study». The Spanish Journal of Psychology, 16, E6.

Serrat, Elisabet; Sidera, Francesc (2018). "Conocimiento emocional y lenguaje». En Desarrollo emocional en los primeros años de vida: debates actuales y retos de futuro (pp. 105-128). Pirámide.

Serrat, Elisabet; Serrano, Jèssica; Amadó, Anna; Sidera, Francesc; Roqueta, Clara; LLOVERAS, SANDRA; BADIA, IRIS (2012). «Training in false-belief understanding in children with language difficulties». Revista de Logopedia, Foniatria y Audiologia, 32(3), 109119. 
Serrat, Elisabet; Sidera, Francesc; Rostan, Carles; Amadó, AnNa (2015). «Adquisició del llenguatge i cognició». Llengua, societat $i$ comunicació= Language, society and communication, $13,30-37$.

Sidera, Francesc; Amadó, ANNA; MARTínez, LAURA (2017). «Influences on facial emotion recognition in deaf children». Journal of Deaf Studies and Deaf Education, 22(2), 164177 .

Sidera, Francesc; Morgan, Gary; Serrat, Elisabet (2020). «Understanding Pretend Emotions in Children Who Are Deaf and Hard of Hearing». The Journal of Deaf Studies and Deaf Education, 25(2), 141-152.

Sidera, Francesc; Serrat, Elisabet; Amadó, AnNa (2014). «Children's understanding of pretend emotions: The role of the vocabulary and the syntax of complementation with cognitive and communicative verbs». L'Anné Psychologique, 114, 231-249.

Siegal, Michael (2008). «Marvelous minds: The discovery of what children know». Oxford, United Kingdom: Oxford University Press.

Skliar, Carlos; Massone, María Ignacia; Veinberg, Silvana (1995). «Deaf children's access to bilingualism and biculturalism». Infancia y Aprendizaje, 18, 69-70, 85-100.

Sprung, Manuel; Münch, Hannah M.; Harris, Paul L.; Ebesutani, Chad; Hofmann, StEFAN G. (2015). "Children's emotion understanding: A meta-analysis of training studies». Developmental Review, 37, 41-65.

Taumoepeau, Mele; Reese, Elaine (2013). «Maternal reminiscing, elaborative talk, and children's theory of mind: An intervention study». First Language, 33(4), 388-410.

Taumoepeau, Mele; Ruffman, Ted (2006). «Mother and infant talk about mental states relates to desire language and emotion understanding». Child Development, 77(2), 465481.

Taumoepeau, Mele; Ruffman, Ted (2008). «Stepping stones to others' minds: Maternal talk relates to child mental state language and emotion understanding at 15, 24, and 33 months». Child Development, 79(2), 284-302.

Tobey, Emily A.; Thal, Donna; Niparko, John K.; Eisenberg, Laurie S.; Quittner, Alexandra L; Wang, Nae-Yuh; CDACI Investigative Team (2013). "Influence of implantation age on school-age language performance in pediatric cochlear implant users». International Journal of Audiology, 52(4), 219-229.

TOMASEllo, Michael (2000). «The social-pragmatic theory of word learning». Pragmatics, 10(4), 401-413.

Tomaszewski, Piotr; Krzysztofiak, Piotr; Moron, Ewelina (2019). «From Sign Language to Spoken Language? A New Discourse of Language Development in Deaf Children». Psychology of Language and Communication, 23(1), 48-84.

TUlLER, LAURICE; DELAGE, HÉLÈNE (2014). «Mild-to-moderate hearing loss and language impairment: How are they linked?» Lingua, 139, 80-101.

WANG, HUizhi; WANG, Yifang; Hu, Yousong (2019). «Emotional understanding in children with a cochlear implant». Journal of Deaf Studies and Deaf Education, 24(2), 65-73.

WANG, YIfANG; SU, YANJIE; YAN, SoNG (2016). «Facial expression recognition in children with cochlear implants and hearing aids». Frontiers in Psychology, 7, 1-6.

Wellman, Henry M.; Peterson, Candida C. (2013). «Deafness, thought bubbles, and theory-of-mind development». Developmental Psychology, 49(12), 2357. 
WELLMAN, HENRY M. (2018). «Theory of mind: The state of the art». European Journal of Developmental Psychology, 15(6), 728-755.

WiefFerink, C. H.; RiefFe, C.; KetelaAR, L.; De Raeve, L.; FriJns, J. H. M. (2013). «Emotion understanding in deaf children with a cochlear implant». Journal of Deaf Studies and Deaf Education, 18(2), 175-186.

Ziv, Margalit; Most, Tova; Cohen, ShiRit (2013). «Understanding of emotions and false beliefs among hearing children versus deaf children». Journal of Deaf Studies and Deaf Education, 18(2), 161-174. 